\title{
Using experience-based co-design to improve inpatient mental health spaces
}

\author{
Zoë Boden, Michael Larkin, Neil Springham
}

\begin{abstract}
Inpatient services are frequently constructed as a topic of concern in research and policy, often in response to service-users' reports that wards are unsafe, boring, and lacking in amenities (Quirk \& Lelliott, 2001). Our research shows that service-users, as well as staff and families, experience inpatient mental health spaces as impermeable, separate and stigmatising, and sometimes uncomfortable, chaotic and unsafe (Fenton et al., 2014; Hickman et al., 2015). Experience-based co-design (EBCD; Bate \& Robert, 2007) is a participatory action research approach to service development, which has been used extensively in physical healthcare (Donetto, Tsianakas \& Robert, 2014), but is only recently being used to improve mental health services (Larkin, Boden \& Newton, 2014). This chapter will draw on EBCD projects from two NHS Mental Health Trusts. These projects brought together service-users, staff and families, alongside Trust management and community staff to co-design improvements to the inpatient wards. Sometimes these improvements were as simple as introducing soft furnishings and better signage, sometimes they were more complex interventions in the culture of the wards, however all the improvements, and perhaps more importantly, the improvement process, allowed service-users and families to feel more welcomed and comforted, and helped staff working in difficult circumstances feel more supported.
\end{abstract}

\section{Inpatient spaces: Challenges in acute mental health services}

Whilst some inpatient mental health services provide pockets of excellence (NICE, 2012), a major UK government report (Crisp, Smith \& Nicholson, 2016) has recently found many under great pressure. Staff are often demoralised and locked into cycles of crisis management, and service-users and families may feel disenfranchised and excluded. Dangerous and chaotic wards, diverse case-mixes, the lack of therapeutic input and the excessive focus on community services at the expense of inpatient settings, all threaten the quality of acute care (Lelliot, Bennett, McGeorge, and Turner, 2006). Service-users (Mind, 2011), families, (Hickman et al., 2015) and staff (Garcia, Kennet, Quarishi \& Durcan, 2005) all agree that acute mental health care is unsatisfactory, frightening and stigmatising. There is even evidence that being hospitalised for a mental health problem can be, in itself, traumatic (Morrison, Bowe, Larkin \& Northand, 1999). This may be unsurprising given the coercive and controlling practices that take place there, including detention, seclusion, restraint and being forcibly compelled to undergo pharmacological treatments, or electro-convulsive therapy.

It is worth questioning how a healthcare environment ever came to be associated with these types of coercive practices. In contemporary society, problems of psychological distress are generally conceptualised as problems of 'mental health'. At times of acute distress, such experiences are subject to psychiatric care, and if the distress involves risk of harm to self or others, then such care is usually provided in an inpatient environment, and the Mental Health Act can be invoked. Currently the majority of service-users on an NHS inpatient ward are there involuntarily (Keown et al., 2016). This view - of what is problematic, who it affects, and how it should be dealt with - is the consequence of a 
series of historical developments as much as it is a consequence of any experiential reality of 'feeling distressed.' In Foucault's (2006/1964) reading, mental health problems first emerged as a category of 'otherness' which needed to be managed alongside a number of other marginal or problematic forms of identity. In his account, people experiencing psychological distress are among several categories of people who may threaten the smooth running of the state. The state therefore, steps in to provide institutional control, meaning the early asylums emerge primarily to control the external environment, rather than for the benefit of those people who found themselves inside. However, it is also notable that distress is medicalised early on in these developments: medical assessment and intervention was developed in tandem with the control and surveillance. As a consequence, inpatient wards have retained some features of both cultures, in an "an uneasy compromise between a general hospital and a prison" (World Health Organisation, 1953). This remains the case for modern wards (Haigh, 2002).

In 1945, there was uncertainty about whether 'mental health' should be situated within the NHS (Kinderman, 2014). If it had not been given to health professionals to meet this need, then acute mental health treatment might have been quite different. It can be helpful to consider how the needs of people in psychological distress might be met from a design perspective. When designing a service or product, three domains are considered: performance, engineering, and aesthetics (Berkun, 2004). Performance concerns whether the product or service works, so designers would likely introduce evidence-based interventions. Engineering involves ensuring those interventions are safe and reliable. Lastly, aesthetics focuses both on the physical environment, such as decoration and furnishing, and on the human and social world, that is how a service or product feels. Performance, engineering and aesthetics must work together. The design process involves testing this, experientially. Designers want to understand the emotional touch-points; how a service user feels when interacting with the service. Problems occur if performance, engineering and aesthetics do not work together or if testing has been insufficient. At first glance, the NHS quality streams map reasonably well onto this design process. Performance is currently covered by clinical effectiveness, engineering by patient safety, and aesthetics by patient experience. However, despite a number of more recent initiatives, such as Enhancing the Healing Environment (Department of Health, 2008), which have attempted to focus on the aesthetics of mental health services, it has taken the scandal highlighted by the Francis report (Mid Staffordshire NHS Foundation Trust, 2013), to instigate a duty to engage with the patient's human experience of their healthcare. The NHS has yet to develop consistent and effective practices which use patient experience data to initiate change (Coulter, Locock, Ziebland \& Calabrese, 2014).

When people are taken to mental health wards, typically in crisis, the aesthetic experience will be substantially different from when a person visits hospital with a physical health problem. Societal views of mental distress, the disruption to the individual's social context, and the subjective experience itself, make the mental health crises unique. The projects and the Experience-based Co-design (EBCD) approach we are going to discuss in this chapter, suggest that key elements of service-users', staff members' and carers' experience in inpatient services (the emotional touchpoints), evoke strong and universal feelings, including terror, shame and alienation. These experiences are not routinely captured by the NHS quality measures, and those admitted to mental health wards do not have the consumer power to vote with their feet - services are commissioned on their behalf and they have little or no opportunity to choose where they are treated. 
In her article on the therapeutic spaces, Fenner (2011) argues that our understanding of the therapeutic relationship should be extended to take into account the material and spatial aspects of the environment in which the relationship occurs. In considering inpatient spaces, the reverse needs to happen; consideration of the environment needs to extend to thinking about the people who occupy these spaces, and how they interact. Spaces are given shape by the practices that are conducted within them; they have functions, atmospheres and cultures. The inpatient ward might be best understood as a 'therapeutic landscape' (Gesler, 1993); a place, expected to be beneficial to wellbeing, where the physical, social and symbolic aspects of the environment, and their cultural and historical context, will elicit particular experiences and meanings for the people who use it (Wood et al., 2015). These subjective experiences and meanings will be contingent upon individuals' and groups' personal attitudes (Coradson, 2005), their emotional experiences, and their memories of other therapeutic landscapes (Wood et al., 2015), for example those encountered in previous admissions.

The literature on psychologically-informed environments literature suggests that spaces can be changed by careful consideration of who resides there, and particular consideration of their psychological and emotional needs (Johnson \& Haigh, 2010). At the most basic level, an inpatient ward needs to be "a good place to be"1 (Haigh, Harrison, Johnson, Paget \& Williams, 2012, p36). In thinking about improving inpatient spaces, it is necessary to include all aspects of the psychosocial environment alongside the physical features. Whilst some mental health hospitals may visually resemble other kinds of hospital, or even office spaces, they are very clearly distinct in terms of their social rules, expectations, practices, affectivity, and culture. Some of these distinctive features are best understood as properties of the spaces themselves (rather than say, as norms or ideas held by any specific person or group). The EBCD approach we now turn to, is promising because, while it explicitly focuses on changing fairly 'concrete' aspects of the environment, it does so via a relational, collaborative mechanism that seems to work - implicitly - to improve psychosocial aspects of the environment as well.

\section{Experience-based Co-design}

Services can be developed from a range of rationales, and the role and source of evidence may vary. In conventional evidence-based practice, a line is presumed to run from acceptability, to efficacy under controlled conditions, and then to effectiveness in naturalistic settings. Yet many interventions supported by high quality RCT evidence, such as family therapy interventions for psychosis, have been difficult to implement in front line practice (Berry \& Haddock, 2008). NICE often over-looks experiential data as suitable evidence, yet understanding the context in which services or interventions happen (as a staff member, service-user or carer) is vital to understanding the local variables which can act as tangential barriers and which may not be apparent in other contexts. To design an effective service or environment, we need to integrate contextbound experiential insights and more traditional sources of (generalised) evidence.

Crisp et al. (2016) recommend that the service design, provision and governance of acute care can be improved by greater collaboration between providers, users and commissioners of inpatient mental health services. EBCD is one such way of working collaboratively to improve health care services. EBCD enables service-users, carers and staff (both ground-level and management) to work together to improve services and

\footnotetext{
${ }^{1}$ The argument which follows is largely focused upon how an existing inpatient environment might be improved, but the processes, of course, can also be used to think about how an alternative forms of 'safe places' might be co-designed.
} 
healthcare environments. EBCD has roots in participatory action research, user-centred design, learning theory and narrative-based approaches to change (Robert, 2013). It was developed for physical health care and was first piloted in a Head and Neck Cancer service (Bate \& Robert, 2007). Subsequently a toolkit was developed (The King's Fund, 2012) to assist local teams in using EBCD in their services.

\section{The EBCD process}

EBCD follows a basic six-step process, and although this has evolved with the literature, most projects in physical healthcare follow this standard sequence (see Fig. 1). Firstly, experiences are gathered from staff, service-users and sometimes carers, via observation and interviews, which are typically filmed. Secondly, "touchpoints" are identified by the project team. These are critical moments experienced in relation to the service. Thirdly these are fed back to the participants, who then prioritise the touchpoints. The penultimate phase is the co-design event, which is the opportunity for different stakeholders to come together and work in small groups to co-design improvements to the service, according to those priorities that have been identified. The final stage is to hold a celebration event to review what has been achieved.

\section{[FIGURE 1 ABOUT HERE]}

\section{Using EBCD to change inpatient mental health spaces}

Mental health contexts are different to physical health ones. Asking service-users to share personal stories of hospitalisation for psychosis, for example, requires different considerations than asking people to talk about the treatment they received for broken bones. In the last few years, a few researchers and clinicians have begun to adapt EBCD for use in mental health contexts. EBCD offers a practical and powerful way of improving mental health services, but it requires a great deal of care to ensure the process does not echo the problems of the services it seeks to improve. We have undertaken EBCD projects in two NHS mental health Trusts in the UK. In Coventry and Warwickshire Mental Health Trust (CWMHT; led by Michael Larkin and Lizzie Newton) the project worked to improve inpatient provision, particularly for young people experiencing early psychosis. At Oxleas Mental Health Trust (led by Neil Springham) the project worked to improve the ward environments in acute adult mental health, with the aim of reducing service-user complaints (Springham \& Robert, 2015). Both projects sought to make positive changes to the inpatient experience, but adapted EBCD in slightly different ways.

\section{[FIGURE TWO ABOUT HERE]}

\section{Developing trust and cooperation: overcoming the challenges}

EBCD is a collaborative approach, and mental health inpatient wards are difficult environments for the development of trust between staff, service-users and carers. The service-user perspective has traditionally been denigrated as lacking in insight, and has 
often been ignored in service planning (Repper and Perkins, 2006). At Oxleas, serviceusers were concerned that what they share in the project could have implications if they needed to return to hospital. The imbalance of power between providers and users of acute inpatient services is heightened in comparison to physical healthcare. Service users may be frightened of service-providers: they have the power to act coercively, and to interpret information provided to them in the light of a 'risk' discourse, which can threaten service-users' liberty and autonomy. Service providers may also sometimes be frightened of service-users: in acute settings, emotions are intensified, and behaviour may seem unpredictable or extreme. There are also very real consequences for staff speaking out in terms of livelihood and career progression. At Oxleas, many staff feared talking freely, in case their feelings were seen as "un-PC". They were worried that any negative feelings that they shared would be deemed indicative of personal incompetence, rather than as systemic, service-level issues. Staff may need reassurance that EBCD is a design project about an improved future, and not an investigation raking over the past or allocating blame - perhaps a far the more common experience of NHS management processes.

Co-operation between groups with different interests and statuses is improved when there is trust between them. Trust in this context might relate particularly to the transparency of each group's motives, to the reliability of the groups (in terms of honouring its commitments), and to the respect that each group is able to show for other group's motives and concerns. In mental health services, and particularly in inpatient care, trust can be threatened by the dual role of the service provider, as both coercive and caring. EBCD contains a number of features that are very helpful for developing trust, and producing effective collaborations.

EBCD invites people to identify their individual concerns, but is predicated on high levels of perspective-taking. Its structured nature means that participants are encouraged to anticipate sharing their experiences with people who have quite different experiences from them, before they are brought face-to-face at the co-design event. Importantly, EBCD treats everyone's experience as equally relevant. All 'stakeholder' groups are consulted and all contribute data that lead to the generation of touchpoints. It ensures that each group has a say in how touchpoints are prioritised. It also invites groups to consider the perspective of others, which is critical. In the 'feedback groups', participants are consulted in the relatively 'safety' of their own stakeholder group. Here there can be considerable reflection on what might be important to the other groups, and allows for a discussion of what it will be like to work with those groups at the 'codesign' stage. Preparation for this is important: groups can discuss how they will work, what they are worried about, and what they hope to gain. Via these processes, EBCD generates consensus and allows for crucial differences in perspective. One coproduction study in a London mental health service measured its success in terms of each party not feeling they had to shift on key positions, but finding recognition of their perspective in the final result (Gillard et al., 2010). The work must proceed with a focus on what everyone agrees to be important, but often there is considerable surprise about how similar everyone's concerns are, once differences have been respected.

\section{The co-design event - opening up new spaces for dialogue}

When bringing together each participant group for the first time, in the co-design event, the aim is to support individuals to express their perspective and to hear the perspectives of others, so that they can collaborate to design improvements to their environment. This has to be carefully panned and managed to ensure that these potentially mistrusting and unequal groups can come together in an atmosphere of 
mutual respect. The aim is to open up a new space, which takes people out of their usual territorial position. The result can be very positive, and can help develop alternative formulations about how to constellate the shared physical and psychosocial environment. In one example, at Oxleas, the co-design process led to the instigation of daily community patient-experience meetings on an inpatient ward. This in turn helped reduce formal complaints by effectively helping staff understand how their behaviour and attitudes were being perceived by service-users (Springham \& Robert, 2015).

The co-design event can be seen as an example of the contact hypothesis (Allport, 1954) in practice. The hypothesis, which has been robustly supported (Pettigrew \& Tropp, 2006), states that intergroup contact will reduce hostility or prejudice. Four conditions are necessary: common goals, intergroup cooperation, support from authorities, and the equal status of parties (Pettigrew \& Tropp, 2005). Well-meaning attempts to bring groups together may actually cause more harm than good if these conditions are not met (see Hewstone \& Swart, 2011). In EBCD, the feedback groups ensure there are common goals. The principle of co-design, implying partnership, encourages cooperation between groups and senior support form management authorities should be sought from the beginning. There are, as has been argued, inequities between service-users and healthcare providers: service-users may be vulnerable due to stigma and distress, and staff members may draw authority from organisational power structures. However, the contact hypothesis literature suggests that, even when there is initially inequality between groups, creating equal status during the contact (the co-design event) is enough to promote positive intergroup attitudes (Schofield \& Eurich-Fulcer, 2001; see Pettigrew \& Tropp, 2005).

The co-design event provides a space that is at least somewhat freed from the normal constraints of the inpatient environment. In this new landscape, service-users, staff and carers are asked to suspend their typical roles, relationships and expectations in order to creatively re-design the inpatient space. The co-design event encourages spontaneous and collaborative interaction. To support participants, and to disrupt the existing culture of the ward, certain practicalities need to be in place. A physically pleasing space (on 'neutral territory', preferably off-site), appropriate refreshments, some easy-toaccess emotional support, a reasonable allotment of time, a clear plan, and achievable goals for the day are elements that go some way towards meeting the requirements for a successful contact intervention. If the event has gone well, on moving back into the inpatient space, participants show an increased capacity to see beyond the categories of 'staff', 'service-user' or 'carer', to take the other's perspective, and to interact more humanely, responsively and flexibly.

This process is more likely to be supported where there is an established and stable group that is committed to pursuing projects of this kind. At Oxleas, this was a preexisting service-user and carer research group ('ResearchNet'). The beneficial outcomes of EBCD in mental health environments, particularly the relational changes, are more likely to be maintained when this type of collaboration can be revisited on a regular basis. Unlike in physical healthcare, where a co-designed change can be implemented and easily maintained, many of the things that make a difference in mental health care are cultural and relational. Changes are hard won, and easily lost without continued attention, due to staff and service-user turnover and the likelihood that people will fall back into ingrained patterns over time. 


\section{Using EBCD safely}

Using EBCD in mental health services requires additional thought. We have adapted EBCD in various ways, so that it can be used successfully and safely in a mental health context. This has involved drawing on research ethics and peer research frameworks to ensure the work is done respectfully and without risk of harm. As shown, mental health services are not viewed favourably by service-users and carers (Mind, 2011) and there are power dynamics that need acknowledgment. Recruitment to an EBCD project needs to be carefully managed. Service-users must not feel coerced into taking part, nor feel they face barriers to participation. Participants must be able to give informed consent at the start, and assent during the project should also be carefully monitored. Some service-users may have experiences that limit their willingness or capacity to engage with particular aspects of an EBCD project, for example being recorded (e.g. due to paranoia) or taking part in group events (e.g. due to social anxiety). Co-design teams must find ways to avoid causing distress and to encourage inclusivity need consideration. There may additionally be practical and social barriers to participants' engagement, such as shift patterns, wellness, discharge plans, childcare, etc. to accommodate.

In standard EBCD, one of the key aspects of the process is the filming of interview footage to be shared at the co-design event. There are a number of potential issues with this in the inpatient mental health context. Service-users may have histories of trauma and abuse, and there is a risk of re-traumatisation in collecting experiential accounts, and in sharing them with others. Issues of confidentiality and anonymity, and consideration of the legacy and ownership of the material must be thought through. Whilst a service-user may be willing to share a distressing account of their experiences during the project, they may change their mind later in their recovery. We would suggest that those leading the co-design projects are cautious in how they collect accounts and what they do with them.

Our projects came up with two different ways to manage these challenges: In the CWMHT project, we audio recorded and transcribed the original experiential accounts, following a typical qualitative research approach. We then asked for service-user and carer volunteers from the feedback groups to create more bespoke short films with us to be shown at the co-design event. We wanted to capture the 'real life' positives and negatives that had been highlighted in the interviews, and give those staff who had not had already heard the findings the chance to get a better understanding of service-user and carer views, but we wanted to ensure this happened in a safe way. We held new interviews with the volunteers that still focused on the volunteers' experiences of inpatient care, but were less open-ended than the original interviews. The aim was to gather filmed material relating solely to the prioritised touchpoints. Each interview lasted approximately half an hour, and each was edited down to a 2-minute clip in the form of a condensed narrative. We were mindful that some of what volunteers said in these interviews might not feel safe to share in front of a large audience, and so we edited out personal details or anything that seemed unnecessarily exposing. We chose extracts that did not feel too controversial, but which linked to the priority areas. For example, a carer talking about how she had been frantic with worry when her son went missing. After many calls, she eventually located him in the local inpatient service. We linked this to our priority area of "communication" because it was clear that a call saying that her son was safe, would have prevented a lot of anxiety. We previewed the clips with the volunteers to make sure that they were still happy for them to be shown. To help the audience, we also added onscreen titles to link the narratives to the priority areas. At Oxleas, initially no one wanted to make films, however once the service-user design group got together to define the bounds of consent and data protection, and 
agreed to make and edit the films themselves, it was decided they would go ahead. Ownership throughout the production of the material ensured that people felt safe. In fact, they felt proud of the films, and saw high levels of commonality between their experiences. Having control over the process, and the support provided by the group, including the chance revisit issues as necessary, helped people to feel they could engage safely and on their own terms.

The co-design event can also become a source of distress for service-users, carers and staff. Meeting with each other can be intimidating and anxiety provoking, but can also be deeply affecting. Great care was taken to prepare service-users, families and staff very carefully for what to expect. We were conscious of the current context of inpatient care in the UK (e.g. Crisp et al., 2016), and that relationships between staff, service-users, and carers may be strained. Although most staff hold positive views towards service-users, there is evidence reporting that some mental health professionals hold negative attitudes toward service-users (see Wahl and Aroesty-Choen, 2010) and can display the same negative stereotypes as the general public (Nordt, Rossler \& Lauber, 2006). As working in inpatient care can be very stressful (e.g. Ward, 2011), there can be widespread demoralisation (Crisp et al., 2016), and many staff can feel pressured by issues such as high bed occupancy, lack of training, and lack of experienced leadership (Garcia et al., 2005). For these reasons, at CWMHT, there was a quiet room and support available for anyone who needed it, regardless of what role they held in the service. In this case, it was a member of staff who made use of these resources.

\section{Making changes in mental health spaces}

In mental health, there is a significant gap between the hopes of service-users and their families, and the nature of services. To a degree, this may be due to the mixed 'carecoercion' model, and the shortage of effective medical interventions for many common difficulties (despite the continued dominance of the medical model). Thus a degree of expectation management is required. Appropriate aims must be established and the remit of the project must be clear. A local co-design project will not rewrite the Mental Health Act, provide more funding for therapists, nor challenge cultural-level stigma, for example. However, it can reasonably aim to improve the environment in which services are delivered, and the way in which they are delivered. It may improve the relationships between the various groups involved. There may also be some anxiety about experiential evidence: in our experience, the issues raised and improved through EBCD do not contradict evidence-based practice. They tend to complement good practice, or enable it to be enacted.

It can be a challenge to make sure that actions planned at the co-design phase are actually implemented. It helps if there is 'ownership' of the project from within the service. It helps too, if there is a group that meets regularly to monitor progress, and if that group contains service-users and families, as well as senior representatives from the host organisation who can make things happen. In both settings, when management were more closely involved, they found it a moving experience. They described how normal reporting structures tended to edit out the experience of staff and users. Sometimes, the presence of an external collaborator, such as a researcher or evaluator can also be helpful. At points in both projects, we found meetings were cancelled due to genuine emergencies on the ward. It was at this point that service users became rather demoralised, as this mirrored their experience of feeling burdensome or undeserving of attention when more demanding things were happening. Likewise it was hard for staff to ignore emergencies to attend EBCD meetings, when they were liable to be blamed if things escalated on the ward. Having protected time set aside for regular meetings is supportive of all parties. 


\section{Conclusions}

Environments dedicated to acute mental healthcare require our attention. They are frequently discomforting, and are often disliked by all parties. However, making changes to mental health inpatient spaces is challenging and requires acknowledgment of the complexity of the environment. Inpatient wards are more than their material and spatial characteristics; they are relational, affective and atmospheric cultures. One way to improve mental health inpatient spaces is through use of the adapted EBCD described in this chapter. This approach is particularly successful when it can be implemented as part of a rolling programme of collaborative improvement work. In this context, EBCD can have a lasting impact on the culture of an inpatient environment, as well making changes at a more concrete level.

Success can be evaluated by monitoring whether the actions agreed at the co-design event have been implemented. Often it is surprisingly easy to think of broad indicators, (for example, number of complaints, or use of coercive measures such as restraint), which could be audited pre- and post- implementation, to judge whether actions have had an effect on the wider environment. There may also be ways of evaluating changes in the relationships between stakeholder groups, depending on the context of the project. Secondly though, projects can lead to unexpected changes at an experiential level for individuals and groups. It is these changes that appear to be the most powerful, but the hardest to evaluate. Qualitative exploration of the impact of EBCD projects would be valuable. The success of EBCD projects is dependent on project leaders having the skill set required, as well as access to the resources they need, and buy-in from senior management and key stakeholders. Competencies with group work, a level of clinical understanding and an 'insider' status are necessary for project leaders.

EBCD for mental health is still in its earliest stages and EBCD more generally is still an emerging field. It is not yet clear what the core elements of the process are or exactly which psychosocial mechanisms are at play. One danger is that EBCD methodology is coopted for use in non-collaborative approaches (Mulvale, Miatello, Hackett \& Mulvale, 2016). However, what has been published about EBCD to date, suggests that it can be a powerful mechanism for service improvement, making services more acceptable to service-users, carers, and staff. EBCD methodology thus has the potential to increase well-being for all concerned. However, challenges encountered in our work adapting EBCD for use in the mental health arena do illustrate the importance of implementing the approach with the right support, resources and care.

\section{Acknowledgements}

With particular thanks to Ami Woods (Oxleas) and Elizabeth Newton (CWMHT), as well as all the participants - service-users, carers and staff.

\section{References}

Allport, G. W. (1954). The nature of prejudice. Reading, MA: Addison-Wesley.

Bate, P., \& Robert, G. (2006). Experience-based design: From redesigning the system around the patient to co-designing services with the patient. Quality and Safety Health Care, 15, 307-310. 
Bate, P., \& Robert, G. (2007). Toward a more user-centric OD: Lessons from the field of experience-based design and a case study. The Journal of Behavioral Science, 43, $41-66$.

Berkun, S. (2004). Programmers, designers and the Brooklyn Bridge. Retrieved from: http://www.scottberkun.com/essays/essay30.htm

Berry, K. \& Haddock, G. (2008). The implementation of the NICE guidelines for schizophrenia: Barriers to the implementation of psychological interventions and recommendations for the future. Psychology and Psychotherapy: Theory, Research and Practice, 81, 419-436.

Coulter, A., Locock, L., Ziebland, S., \& Calabrese, J. (2014). Collecting data on patient experience is not enough: they must be used to improve care. British Medical Journal, 348, g2225.

Crisp, N., Smith, G. and Nicholson, K. (Eds.) (2016). Old Problems, New Solutions Improving Acute Psychiatric Care for Adults in England. The Commission on Acute Adult Psychiatric Care. Retrieved from: http://media.wix.com/ugd/0e662e_aaca63ae4737410e9e2873dfde849841.pdf

Department of Health (2004). Improving the patient experience: sharing success in mental health and learning disabilities: The King's Fund's Enhancing the Healing Environment Programme 2004-2008. London: TSO.

Donetto, S., Tsianakas, V., \& Robert, G. (2014). Using experience-based Co-design (EBCD) to improve the quality of healthcare: Mapping where we are now and establishing future directions. London: King's College London.

Fenton, K., Larkin, M., Boden, Z., Thompson, J., Hickman, G., \& Newton, E. (2014). The experiential impact of hospitalisation in early psychosis: Service-user accounts of inpatient environments. Health and Place, 30, 234-241.

Foucault, M. (2006). Madness and Civilization. A history of insanity in the age of reason. London: Vintage Books. (Original work published 1964.)

Garcia, I., Kennet, C., Quarishi, M., \& Durcan, G. (2005). Acute Care 2004: A national survey of adult psychiatric wards in England. London: The Sainsbury Centre for Mental Health.

Gesler, W., 1993. Therapeutic landscapes - theory and a case study of Epidauros, Greece. Environment and Planning D: Society and Space, 11 (2), 171-189.

Gillard S., Turner K., Lovell K., Norton, K., Clarke, T., Addicott, R., McGivern, G., \& Ferlie, E. (2010). "Staying native": coproduction in mental health services research. International Journal of Public Sector Management 23(6), 567-77.

Haigh, R. (2002). Acute wards: problems and solutions. Modern millieux: therapeutic community solutions to acute ward problems. Psychiatric Bulletin, 26, 380-382.

Haigh, R., Harrison, T., Johnson, R., Paget, S., Williams, S., (2012). Psychologically informed environments and the "Enabling Environments" initiative. Housing, Care and Support, 15(1), 34-42.

Hewstone, M., \& Swart, H. (2011). Fifty-odd years of inter-group contact: From hypothesis to integrated theory. British Journal of Social Psychology, 50, 374386. 
Hickman, G., Newton, E., Boden, Z., Fenton, K., Thompson, J., \& Larkin, M. (2015). The experiential impact of hospitalisation on families of young people with early psychosis. Clinical Child Psychology and Psychiatry, 21(1), 145-155.

Hummelvoll, J. K., \& Severinsson, E. (2001). Coping with everyday reality: Mental health professionals' reflections on the care provided in an acute psychiatric ward. Australian and New Zealand Journal of Mental Health Nursing, 10, 156-166.

Johnson, R. \& Haigh, R. (2010). Social psychiatry and social policy for the 21st century new concepts for new needs: the 'psychologically-informed environment', Mental Health and Social Inclusion, 14(4), 30-35.

Keown, P., McBride, O., Twigg, L., Crepaz-Keay, D., Cyhlarova, E., Parsons, H., Scott, J., Bhui, K., \& Weich , S. (2016). Rates of voluntary and compulsory psychiatric inpatient treatment in England: an ecological study investigating associations with deprivation and demographics. The British Journal of Psychiatry, 209(2) 157-161.

Kinderman, P. (2014). A prescription for psychiatry: why we need a whole new approach to mental health and wellbeing. London: Palgrave Macmillan.

Lelliot, P., Bennett, H., McGeorge, M., \& Turner, T. (2006). Accreditation of acute inpatient mental health services. Psychiatric Bulletin, 30, 361-363.

Mid Staffordshire NHS Foundation Trust (2013). Report of the Mid Staffordshire NHS Foundation Trust Public Inquiry. London: TSO.

Mind. (2011). Listening to experience: An independent inquiry into acute and crisis mental healthcare. London: Author.

Morrison, A., Bowe, S., Larkin, W., \& Nothard, S. (1999). The psychological impact of psychiatric admission: Some preliminary findings. The Journal of Nervous and Mental Disease, 187, 250-253.

Mulvale, A., Miatello, A., Hackett, C., and Mulvale, G. (2016). Applying experience-based co-design with vulnerable populations: Lessons from a systematic review of methods to involve patients, families and service providers in child and youth mental health service improvement, Patient Experience Journal, 3(1), 117-129.

National Institute for Health and Care Excellence. (2012). Service user experience in adult mental health: NICE guidance on improving the experience of care for people using adult NHS mental health services. London: The British Psychological Society \& The Royal College of Psychiatrists.

Nordt, C., Rossler, W., \& Lauber, C. (2006). Attitudes of mental health professionals toward people with schizophrenia and major depression. Schizophrenia Bulletin, $32,709-714$.

Pettigrew, T. F., \& Tropp, L. R. (2005). Allport's Intergroup contact hypothesis: Its history and influence. In J. F. Doviio, P. Glick, \& L. A. Budman (Eds.), On the nature of prejudice: Fifty years after Allport (pp. 262-277). Oxford, UK: Blackwell.

Pettigrew, T. F., \& Tropp, L. R. (2006). A meta-analytic test of intergroup contact theory. Journal of Personality and Social Psychology, 90, 751-783.

Robert, G. (2013). Participatory action research: Using experience-based co-design (EBCD) to improve the quality of health care services. In S. Ziebland, J. Calabrese, 
A. Coulter, \& L. Locock (Eds.), Understanding and using experiences of health and illness (p. 138-149). Oxford, UK: Oxford University Press.

The King's Fund. (2012). Experience-based co-design toolkit. Retrieved from http://www.kingsfund.org.uk/projects/ebcd

Schofield, J. W., \& Eurich-Fulcer, R. (2001). When and how school desegregation improves intergroup relations. In R. Brown \& S. L. Gaetner (Eds.), The Blackwell handbook of social psychology: Intergroup processes (pp. 475-494). Malden, MA: Blackwell.

Springham, N., \& Robert, G. (2015). Experience based co-design reduces formal complaints on an acute mental health ward. British Medical Journal Quality Reports, 4(1), doi:10.1136/bmjquality.u209153.w3970.

Wahl, O., \& Aroesty-Cohen, E. (2010). Attitudes of mental health professionals about mental illness: A review of the recent literature. Journal of Community Psychology, 38(1), 49-62.

Ward, L. (2011). Mental health nursing and stress: Maintaining balance. International Journal of Mental Health Nursing, 20, 77-85.

World Health Organisation (1953). Expert Committee on Mental Health: 3rd Report. Geneva: WHO.

\section{FIGURES}

\section{The standard EBCD process:}

- gathering experiences from staff, then service uses and carers, via observation and interviews (which are often filmed)

- identifying 'touchpoints'; critical moments experiences in relation to the service

- feeding back these touchpoints to project participants

- the prioritising of the touchpoints by the project participants

- bringing everyone together in a co-design event, which they work in small groups to co-design improvements to the service according to the priorities identified

- holding a celebration event to all involved to review what has been achieved

(Donetto et al., 2014; Robert, 2013)

Figure 1. The standard EBCD process 


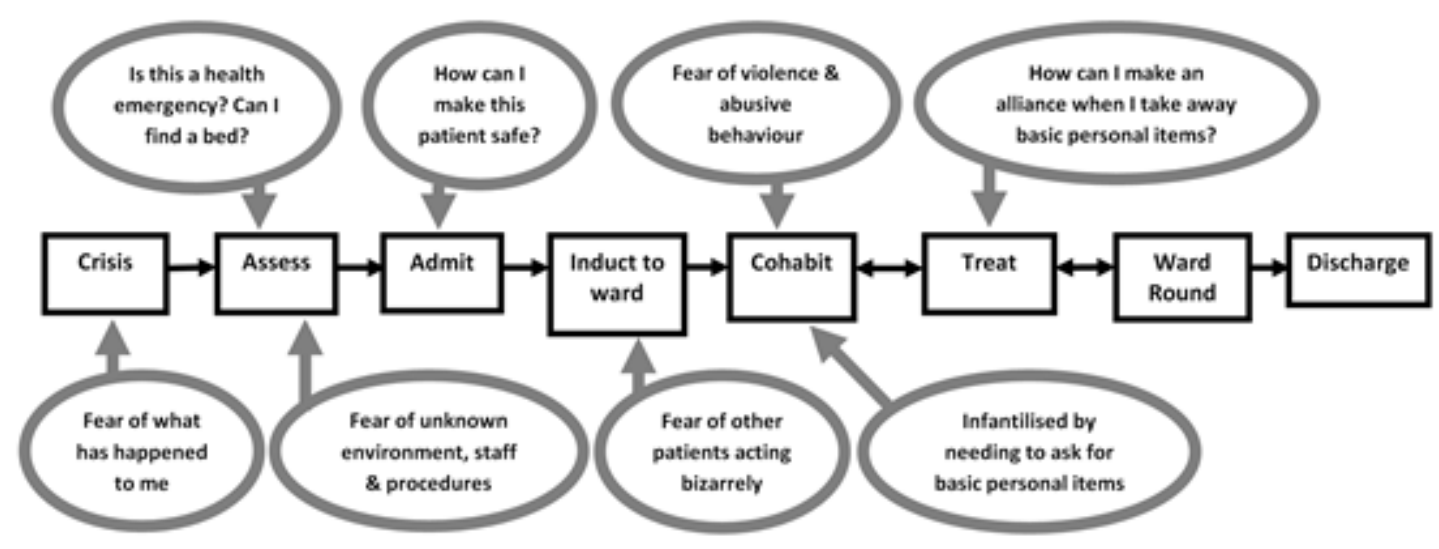

Figure 2. Generating touchpoints in the Oxleas project. The sequence of clinical procedures is shown as squares. Emotional touchpoints are shown as circles, with staff experience shown above and service-user experience below.

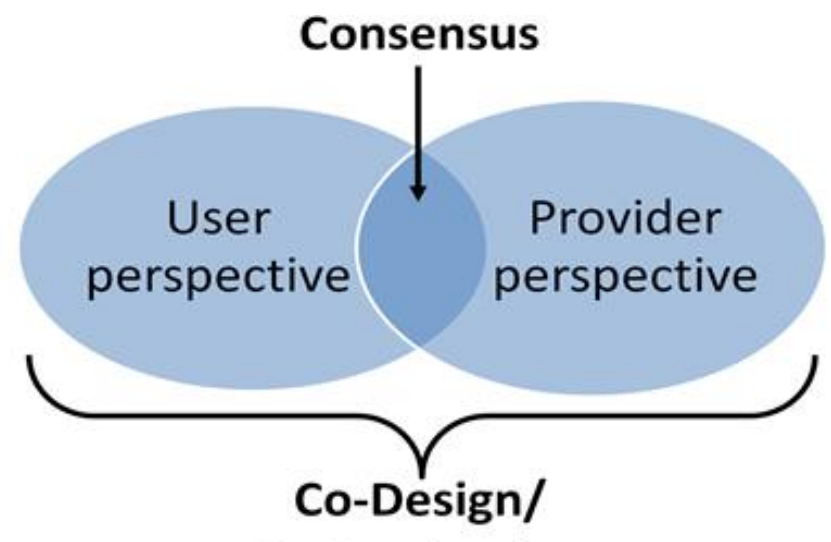

\section{Co-Production}

Figure 3. The differentiation of consensus versus co-production methodologies, where differences of perspective are utilised alongside points of agreement. 